\title{
Fatal hemothorax after thoracic epidural procedure for postoperative pain control in elderly esophageal cancer patient
}

\author{
Jong-Myeon Hong, Dohun Kim, Si-Wook Kim \\ Department of Thoracic and Cardiovascular Surgery, College of Medicine, Chungbuk National University, Chungbuk National University Hospital, \\ Cheongju, South Korea \\ Correspondence to: Jong-Myeon Hong. Department of Thoracic and Cardiovascular Surgery, College of Medicine, Chungbuk National University, \\ Chungbuk National University Hospital, 1 Chungdae-ro, Seowon-gu, Cheongju 362-763, South Korea. Email: hongjm1@gmail.com.
}

Submitted May 11, 2018. Accepted for publication Jun 28, 2018.

doi: $10.21037 /$ jtd.2018.07.24

View this article at: http://dx.doi.org/10.21037/jtd.2018.07.24

\section{Introduction}

Postoperative epidural analgesia after thoracotomy has been one of the most effective methods for relief of the wound pain. But, several minor complications have been alleged related to this procedure including minor hemothorax. We report here a case of catastrophic complication of the thoracic epidural catherization in an elderly patient who underwent Ivor Lewis operation because of the malignant esophageal cancer.

\section{Case presentation}

Modified Ivor Lewis operation was done in an 82-year-old man due to T2N0M0 squamous cell carcinoma using hybrid laparoscopic procedure followed by thoracic esophagectomy and esophagogastrostomy using $25 \mathrm{~mm}$ Auto Stapler through standard right posterolateral thoracotomy. The patient had been well recovered without any specific complication until postoperative \# 9th day except thoracotomy wound pain disturbing night sleep. He had been suffered thoracotomy wound pain, though strong analgesics including intravenous and oral medications. We consulted to an anesthesiologist for pain control, and the patient got the schedule for epidural catheter insertion on postoperative \# 9th day. Eight hours after the procedure, the patient felt chest discomfort with dyspnea, and the following chest X-ray showed lots of hemothorax at right pleural cavity with deviating the heart to the left side (Figure 1). He got respiratory arrest during chest tube management, and cardiopulmonary resuscitation (CPR) was immediately done from the thoracic surgeon on site for over 30 minutes, then

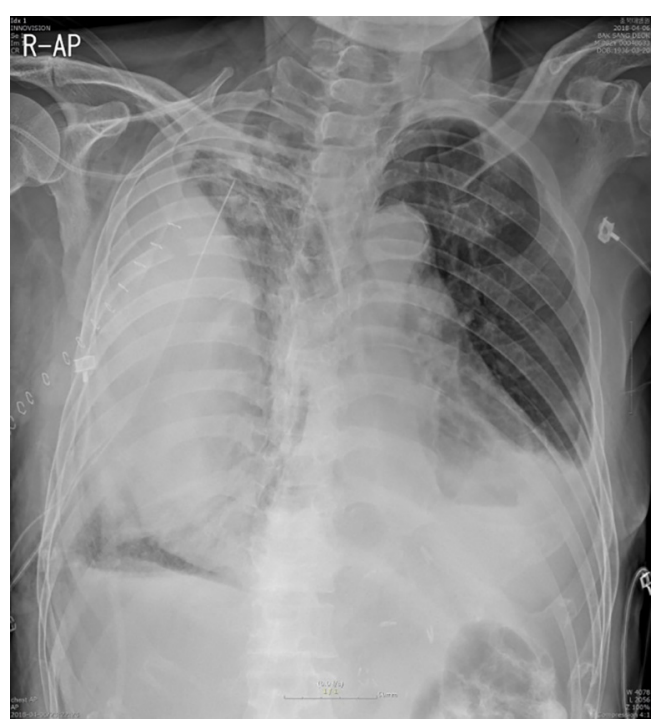

Figure 1 Eight hours after thoracic epidural procedure, chest X-ray showed large amount of right hemothorax with cardiac shifting to left side.

his heart function was returned. After moving to intensive care unit (ICU), another cardiac arrest was happened, and the heart was returned back after CPR, we set up the arteriovenous typed extracorporeal membrane oxygenation (ECMO) at ICU immediately because of the unevacuated huge amount of hemothorax caused to cardiac tamponade. Immediately after ECMO starting, we performed emergent operation, and evacuated lots of hematoma from the right pleural cavity with hemostasis of the small amount of oozing 


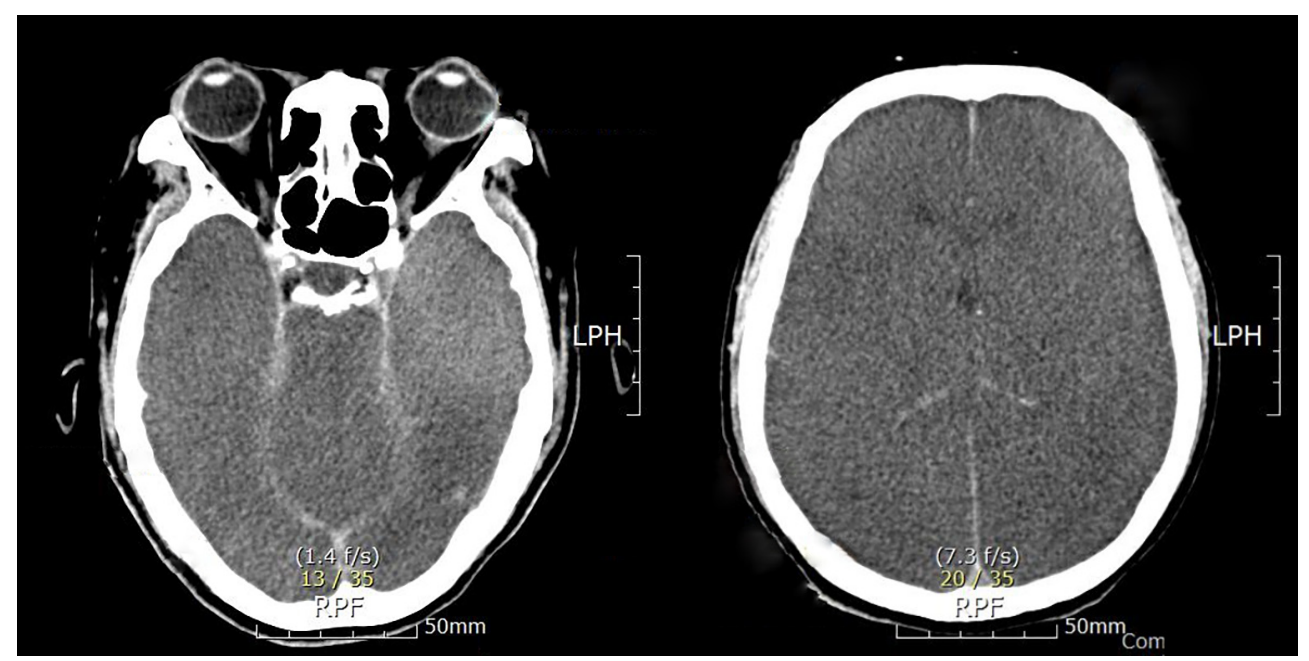

Figure 2 Two days after cardiac massage, the brain CT showed severely generalized ischemic damage of the whole brain. CT, computed tomography.

from the adhesive mediastinal pleurae which we could not find any specific bleeding point except torn intercostal arterial bleeding from the previous thoracotomy wound space. On postoperative \# 10th day we performed brain computed tomography (CT) due to delayed wake-up from the general anesthesia with no response of the neurological test, the CT showed generalized brain swelling which meant severe ischemic damage (Figure 2). The patient's heart stopped on postoperative \# 15th day without any further management.

Ethical approval was obtained from our hospital institutional review boards (IRB FILE No. 2018-06-002), and the patient signed the written informed consent.

\section{Discussion}

Several complications after thoracic epidural catheterization have been reported, such as epidural hematoma, dural puncture, nerve injuries, intrapleural catheter penetration, pneumothorax, and hemothorax (1-5). Among them, injuries to the intercostal vessels could cause the patient to a serious condition because of the catastrophic hemothorax like this elderly patient. The inappropriately directed epidural puncture can cause the injury of the intercostal arteries or veins. Amagasa $e t$ al. recommended to prevent this kind of complications: (I) don't repeat needle puncture into a single intervertebral space; (II) avoid deeply advancing the needle; (III) make sure the patient proper position; (IV) use transparent drape; and (V) median approach if possible (2).
Catastrophic result occurred due to the large amount of hemothorax to cardiac tamponade after epidural procedure, caused by unintended puncture of the intercostal vessels. We should be kept in mind that this kind of fatal hemothorax could be complicated after thoracic epidural procedure.

\section{Acknowledgements}

None.

\section{Footnote}

Conflicts of Interest: The authors have no conflicts of interest to declare.

Ethical Statement: Ethical approval was obtained from our hospital institutional review boards (IRB FILE No. 201806-002), and the patient signed the written informed consent.

\section{References}

1. Sundary MT. Epidural catheter misplaced into the thoracic cavity: Utilized to provide interpleural analgesia. Anesth Essays Res 2015;9:121-3.

2. Amagasa S, Igarashi A, Yokoo N, et al. Intercostal bleeding that developed during thoracic epidural catherization. J Anesth 2008;22:197-8. 
3. Inoue S, Nishimine N, Furuya H. Unintended intrapleural insertion of an epidural catheter: should we remove it or leave it in situ to provide perioperative analgesia? Anesth Analg 2005;100:266-8.

4. Grieve PP, Whitta RK. Pleural puncture: an unusual

Cite this article as: Hong JM, Kim D, Kim SW. Fatal hemothorax after thoracic epidural procedure for postoperative pain control in elderly esophageal cancer patient. J Thorac Dis 2018;10(8):E643-E645. doi: 10.21037/jtd.2018.07.24 complication of a thoracic epidural. Anaesth Intensive Care 2004;32:113-6.

5. Iida Y, Kashimoto S, Matsukawa T, et al. A hemothorax after thoracic epidural anesthesia. J Clin Anesth 1994;6:505-7. 